\title{
Comparison of Different Integral Performance Criteria for Optimal Hydro Generator Governor Tuning with a Particle Swarm Optimization Algorithm
}

\author{
Hongqing Fang ${ }^{1}$, Long $\mathrm{Chen}^{2}$, and Zuyi Shen ${ }^{2}$ \\ ${ }^{1}$ College of Electrical Engineering, Hohai University, Nanjing, 210098 Jiangsu, P.R. China \\ fanghongqing@sohu.com \\ ${ }^{2}$ College of Water Conservancy \& Hydropower Engineering, Hohai University, Nanjing, \\ 210098 Jiangsu, P.R. China \\ \{cdalong, richardshen\}@hhu.edu.cn
}

\begin{abstract}
In this paper, the particle swarm optimization (PSO) algorithm with constriction factor approach (CFA) is proposed to optimal hydro generators governor Proportional-Integral-Derivative (PID) gains for small hydraulic transients. And four different integral performance criteria of turbine speed deviation such as integrated absolute error (IAE), integral of time weighted absolute value of error (ITAE), integral of squared error (ISE) and integral of time weighted squared error (ITSE) have been taken as fitness function respectively, and the differences have been investigated and compared. A step speed disturbance test on no-load operation has been performed. The digital simulation results show that the proposed PSO method has stable convergence characteristic and good computational ability, and it can effectively optimal hydro generators governor PID gains. And the dynamic performance of hydro generators governor system for small hydraulic transients is much better if ITAE criterion is applied as fitness function.
\end{abstract}

Keywords: Particle swarm optimization; Constriction factor approach; Integral performance criteria; Hydro generators governor; PID tuning.

\section{Introduction}

In modern hydroelectric power plants, conventional PID controller is widely applied in hydro generators governor systems [1]. There are any methods have been reported for improving the setting performance of hydro generators governor system parameters, such as simplex method, orthogonal test method, genetic algorithm (GA) and so on [2]. However, these methods have different disadvantages. Particle swarm optimization (PSO) is characterized as a simple concept, easy to implement, and computationally efficient [3]. In fact, PSO has obtained more and more attentions in the researching areas of electric power systems [4]-[5]. In this paper, the PSO algorithm with constriction factor approach (CFA-PSO) [6] is proposed to optimal hydro generators governor PID gains for small hydraulic transients. And four different integral performance criteria of turbine speed deviation such as IAE, ITAE, ISE and ITSE have been taken as fitness function respectively, and the differences have been investigated and compared. 


\section{Hydro Generators Governor System}

The typical hydro generators governor system is shown in Fig. 1, which consists of PID controller, electro-hydraulic servo system, hydro turbine system, generator and load. The definitions of the parameters, in Fig. 1, could be found in [1].

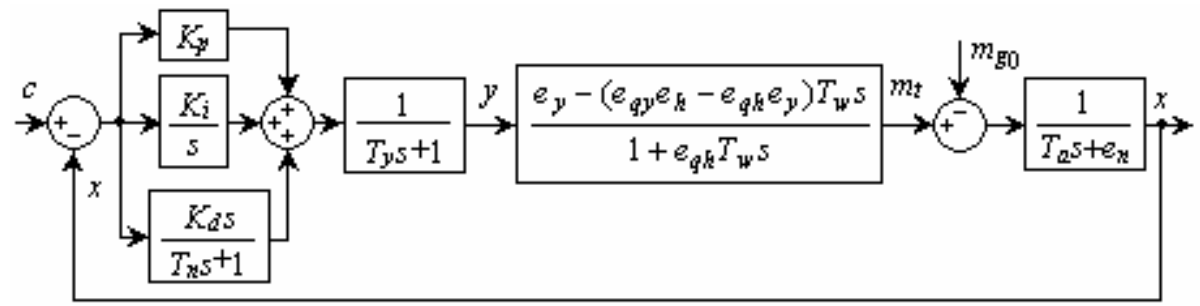

Fig. 1. Typical hydro generators governor system

\section{Implementation of a PSO-PID Controller for Hydro Generators Governor System}

The velocity of the $k$ th dimension of the $i$ th particle is updated in current iteration cycle $(t+1)$ in CFA-PSO can be expressed as:

$$
V_{i},{ }_{k}(t+1)=\chi\left(V_{i},{ }_{k}(t)+c_{1} r_{1}\left(P_{i},{ }_{k}(t)-X_{i},{ }_{k}(t)\right)+c_{2} r_{2}\left(P_{g},{ }_{k}(t)-X_{i},{ }_{k}(t)\right)\right)
$$

And the position of the $k$ th dimension of the $i$ th particle is updated as:

$$
X_{i, k}(t+1)=X_{i, k}(t)+V_{i},{ }_{k}(t+1)
$$

The definitions of the parameters of CFA-PSO could be found in [6]. Usually, $c_{1}$ and $c_{2}$ are set as $c_{1}=c_{2}=2.05$, thus, $\chi=0.729$.

Table 1. Parameters for hydro generators governor system

\begin{tabular}{cccccccc}
\hline$T_{y}$ & $T_{a}$ & $T_{w}$ & $e_{y}$ & $e_{q y}$ & $e_{h}$ & $e_{q h}$ & $e_{n}$ \\
\hline 0.1 & 6.0 & 1.5 & 1.0 & 1.0 & 1.5 & 0.5 & 1.5 \\
\hline
\end{tabular}

A PID controller optimized with the CFA-PSO algorithm was developed, which was called PSO-PID controller. The integral performance criteria (IAE, or ITAE, or ISE, or ITSE criteria) of turbine speed deviation $x$ must be minimized by CFA-PSO algorithm. The CFA-PSO algorithm for the PID gains optimizing was implemented using Microsoft Visual Basic 6.0 programming language on a PC with Intel Pentium III $1 \mathrm{GHz}$ processor. The population size of CFA-PSO algorithm is 15 and the maximum allowable number of iterations is 30 . The data of hydro generators governor system are given in Table 1, the time step of the simulation for small hydraulic transients is $0.02 \mathrm{sec}$, the 
simulation is done for $30 \mathrm{sec}$, and the lower and upper bounds of PID gains are all set as $[0,10]$. The optimizing processes have been done off-line.
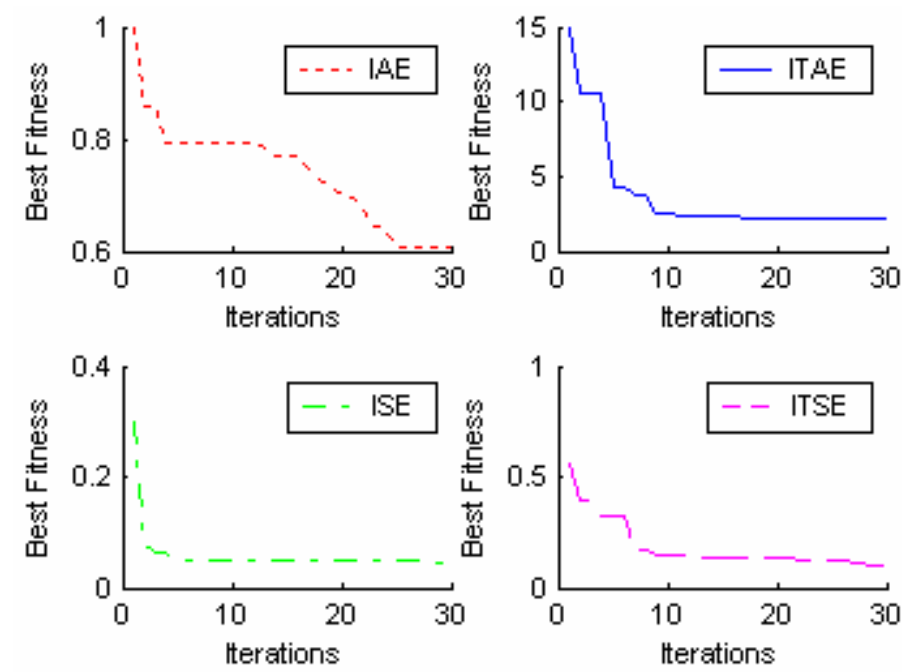

Fig. 2. Best fitness convergence

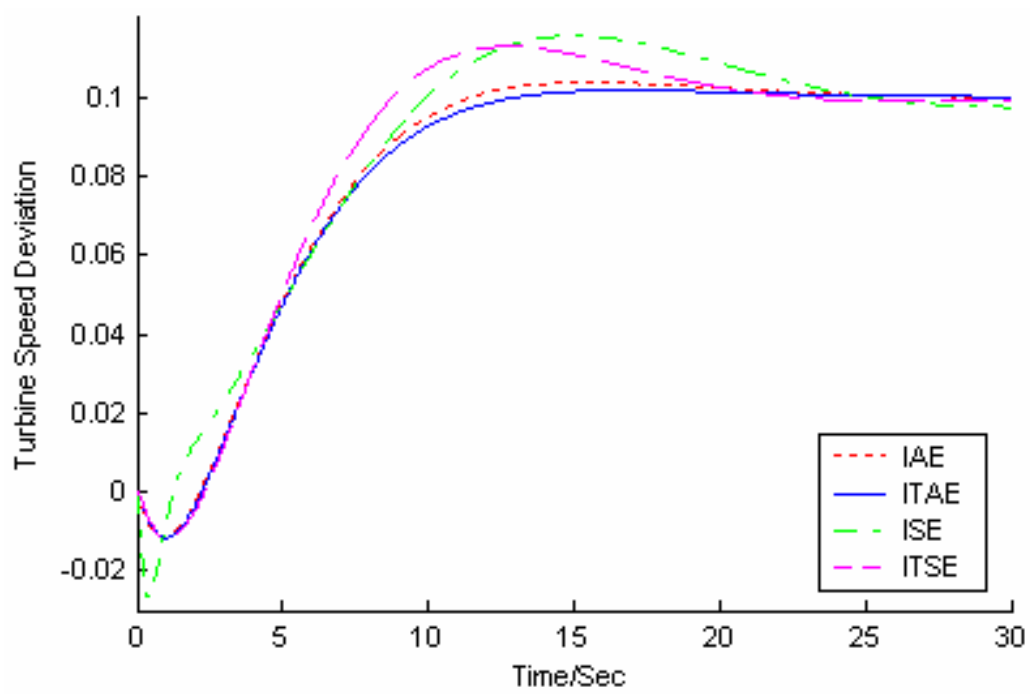

Fig. 3. Speed response to a $10 \%$ step increasing in turbine speed set point

A $10 \%$ step turbine speed deviation increasing was applied to the turbine speed reference, i.e., $c=0.1$. Since all the four integral performance criteria of turbine speed deviation $x$ have been taken as fitness function respectively, thus, there have four different optimal PID gains sets, shown in Table 2. From the simulations results, it 
could be found that if ITAE criterion was taken as fitness function, hydro generators governor system will get the smallest overshoot, i.e., $M_{p}=0.102$, and the shortest settling time, i.e., $t_{s}=11.717 \mathrm{sec}$.The traces of the best fitness convergence of the four integral performance criteria are shown in Fig. 2. It could be found that all of the optimizing processes have downward trends and are convergence in not more than 30 iterations. The fastest one is ITAE criterion was taken as fitness function, which is not more than 16 iterations, and the slowest one is ITSE criteria was taken as fitness function, which is not more than 29 iterations. And Fig. 3 shows the turbine speed deviation response traces using the four different optimal PID gains sets. It is clear that the hydro generators governor system has the best dynamic performance if ITAE criterion was applied as fitness function than that of IAE, ISE and ITSE criterion.

Table 2. Results for a 10\% step turbine speed increasing

\begin{tabular}{cccccccc}
\hline Criteria & $K_{p}$ & $K_{i}$ & $K_{d}$ & $T_{n}$ & $M_{p}$ & $t_{s}$ & $J$ \\
\hline$J_{\text {IAE }}$ & 0.921 & 0.279 & 0.066 & 0.007 & 0.104 & 20.735 & 0.606 \\
$J_{\text {ITAE }}$ & 0.933 & 0.263 & 0.0 & 0.0 & 0.102 & 11.717 & 2.228 \\
$J_{\text {ISE }}$ & 0.741 & 0.356 & 0.946 & 0.095 & 0.117 & 33.110 & 0.049 \\
$J_{\text {ITSE }}$ & 0.895 & 0.340 & 0.0 & 0.0 & 0.113 & 20.455 & 0.109 \\
\hline
\end{tabular}

\section{Conclusion}

This paper, has shown the effectively design method for determining the PID gains for hydro generators governor system applying CFA-PSO algorithm and it is clear that the ITAE criterion is most suitable for the application as fitness function.

\section{References}

1. IEEE Committee Report: Hydraulic turbine and turbine control models for system dynamic studies. IEEE Trans. Power Systems. 1(1992) 167-178

2. Li Z., and Malik O. P.: An orthogonal test approach based control parameter optimization and its application to a hydro-turbine governor. IEEE Trans. Energy Conversion, 4(1997) 388-393

3. Kennedy J., and Eberhart R.: Particle swarm optimization. IEEE Int. Conf. Neural Networks. Purth, Australia, (1995) 1942-1948

4. Abido M. A.: Optimal design of power-system stabilizers using particle swarm optimization. IEEE Trans. Energy Conversion. 3(2002) 406-413

5. Gaing Z. L.: Particle swarm optimization to solving the economic dispatch considering the generator constraints. IEEE Trans. Power Systems. 3(2003) 1187-1195

6. Eberhart R. C. and Shi Y.: Comparing inertia weights and constriction factors in particle swarm optimization. 2000 Congress on Evolutionary Computation. San Diego, (2000) $84-88$ 